\title{
Collision of Shock Waves in Einstein-Maxwell Theory with a Cosmological Constant: A Special Solution
}

\author{
C. Barrabès* \\ Laboratoire de Mathématiques et Physique Théorique, \\ CNRS/UMR 7350, Fédération Denis Poisson CNRS/FR2964, \\ Université F. Rabelais, 37200 TOURS, France \\ and \\ P. A. Hogan ${ }^{\dagger}$ \\ School of Physics, \\ University College Dublin, Belfield, Dublin 4, Ireland \\ PACS numbers: 04.40.Nr, 04.30.Nk
}

\begin{abstract}
Post-collision space-times of the Cartesian product form $M^{\prime} \times M^{\prime \prime}$, where $M^{\prime}$ and $M^{\prime \prime}$ are 2-dimensional manifolds, are known with $M^{\prime}$ and $M^{\prime \prime}$ having constant curvatures of equal and opposite sign (for the collision of electromagnetic shock waves) or of the same sign (for the collision of gravitational shock waves). We construct here a new explicit post-collision solution of the Einstein-Maxwell vacuum field equations with a cosmological constant for which $M^{\prime}$ has constant (non-zero) curvature and $M^{\prime \prime}$ has zero curvature.
\end{abstract}

\footnotetext{
${ }^{*}$ E-mail : barrabes@celfi.phys.univ-tours.fr

${ }^{\dagger}$ E-mail : peter.hogan@ucd.ie
} 


\section{Introduction}

The space-time following the head-on collision of two homogeneous, plane, electromagnetic shock waves was found by Bell and Szekeres [1] and is a solution of the vacuum Einstein-Maxwell field equations. The metric tensor is that of a Cartesian product of two 2-dimensional manifolds of equal but opposite sign constant curvatures and is the Bertotti-Robinson ([2], [3]) spacetime. Recently we have shown ([5], 6]) that the Nariai-Bertotti ([2], [7]) space-time, with metric that of a Cartesian product of two 2-dimensional manifolds of equal constant curvatures, coincides with the space-time following the head-on collision of two homogeneous, plane, gravitational shock waves and is a solution of Einstein's vacuum field equations with a cosmological constant. We construct here a metric for a space-time which is a Cartesian product of two 2-dimensional manifolds, one having non-zero constant curvature and one having zero curvature, and show that the metric is: (I) that of the post collision region of space-time following the head-on collision of two plane light-like signals each consisting of combined gravitational and electromagnetic shock waves, one signal specified by a real parameter $a$ and the second signal specified by a real parameter $b$ and (II) is a solution of the vacuum Einstein-Maxwell field equations with a cosmological constant $\Lambda=2 a b$. The appearance of a cosmological constant term on the left hand side of the Einstein field equations is equivalent to the appearance of an energy-momentum-stress tensor for a perfect fluid for which the sum of the matter proper density and the isotropic pressure vanishes. Thus our spacetime consists of an anti-collision region which is vacuum and a post-collision region which is non-vacuum in this sense. Vacuum and non-vacuum regions of space-time are familiar from solving the field equations for so-called interior and exterior solutions.

\section{Cartesian Product Space-Time}

We consider a pseudo-Riemannian space-time $M$ of the form $M=M^{\prime} \times M^{\prime \prime}$ where $M^{\prime}$ is a 2-dimensional manifold of non-zero constant curvature and $M^{\prime \prime}$ is a 2-dimensional flat manifold. So that the 4-dimensional manifold $M$ has the correct Lorentzian signature we consider the two cases in which (i) $M^{\prime}$ is pseudo-Riemannian and $M^{\prime \prime}$ is Riemannian and (ii) $M^{\prime}$ is Riemannian and $M^{\prime \prime}$ is pseudo-Riemannian. In either case take $\xi, x$ as local coordinates on $M^{\prime}$ and $\eta, y$ as local coordinates on $M^{\prime \prime}$. With $a, b$ real constants we take $a b<0$ for case (i) and write the line element of $M$ as

$$
d s^{2}=d \xi^{2}-\cos ^{2}(2 \sqrt{-a b} \xi) d x^{2}-d \eta^{2}-d y^{2} .
$$


In terms of the basis 1 -forms $\vartheta^{1}=d \xi$ and $\vartheta^{2}=\cos (2 \sqrt{-a b} \xi) d x$ the single non-vanishing Riemann curvature tensor component on the dyad defined by this basis, for the manifold $M^{\prime}$, is

$$
R_{1212}=4 a b,
$$

indicating that the pseudo-Riemannian manifold $M^{\prime}$ has non-zero constant Riemannian curvature (see, for example, 44) $-4 a b>0$. Clearly the manifold $M^{\prime \prime}$ is Riemannian and flat. For case (ii) we take $a b>0$ and write the line element of $M$ as

$$
d s^{2}=-d \xi^{2}-\cos ^{2}(2 \sqrt{a b} \xi) d x^{2}+d \eta^{2}-d y^{2} .
$$

Now $M^{\prime}$ is Riemannian. In terms of the basis 1 -forms $\vartheta^{1}=d \xi$ and $\vartheta^{2}=$ $\cos (2 \sqrt{a b} \xi) d x$ the non-vanishing component of the Riemann curvature tensor for $M^{\prime}$, on the dyad defined by the basis 1 -forms, is

$$
R_{1212}=-4 a b
$$

indicating that the Riemannian manifold $M^{\prime}$ has non-zero Gaussian curvature $K=-R_{1212}=4 a b>0$. In this case the manifold $M^{\prime \prime}$ is pseudoRiemannian and flat. Now for case (i) make the transformation

$$
\xi=\frac{a u-b v}{\sqrt{-2 a b}}, \quad \eta=\frac{a u+b v}{\sqrt{-2 a b}},
$$

while for case (ii) make the transformation

$$
\xi=\frac{a u-b v}{\sqrt{2 a b}}, \quad \eta=\frac{a u+b v}{\sqrt{2 a b}} .
$$

In both cases the line elements (2.1) and (2.3) become

$$
d s^{2}=-\cos ^{2}\{\sqrt{2}(a u-b v)\} d x^{2}-d y^{2}+2 d u d v .
$$

We can write this line element in the form

$$
d s^{2}=-\left(\vartheta^{1}\right)^{2}-\left(\vartheta^{2}\right)^{2}+2 \vartheta^{3} \vartheta^{4}=g_{a b} \vartheta^{a} \vartheta^{b},
$$

with the basis 1 -forms given, for example, by $\vartheta^{1}=\cos \{\sqrt{2}(a u-b v)\} d x, \vartheta^{2}=$ $d y, \vartheta^{3}=d v, \vartheta^{4}=d u$. Thus the constants $g_{a b}$ are the components of the metric tensor on the half-null tetrad defined via the basis 1 -forms. The components $R_{a b}$ of the Ricci tensor on this tetrad vanish except for

$$
R_{11}=-4 a b, \quad R_{33}=-2 b^{2}, \quad R_{34}=2 a b, \quad R_{44}=-2 a^{2} .
$$


With

$$
F=\frac{1}{2} F_{a b} \vartheta^{a} \wedge \vartheta^{b}=a \vartheta^{1} \wedge \vartheta^{4}+b \vartheta^{3} \wedge \vartheta^{1},
$$

and $\Lambda=2 a b$ we have here a solution of the Einstein-Maxwell vacuum field equations with a cosmological constant:

$$
R_{a b}=\Lambda g_{a b}+2 E_{a b},
$$

and

$$
d F=0=d^{*} F,
$$

where $d$ denotes the exterior derivative, ${ }^{*} F=a \vartheta^{2} \wedge \vartheta^{4}+b \vartheta^{2} \wedge \vartheta^{3}$ is the Hodge dual of the Maxwell 2-form (2.10) with components $F_{a b}$ on the tetrad given by (2.10) and $E_{a b}=F_{a c} F_{b}{ }^{c}-\frac{1}{4} g_{a b} F_{c d} F^{c d}$ is the electromagnetic energymomentum tensor. Tetrad indices are raised with $g^{a b}$ where $g^{a b} g_{b c}=\delta_{c}^{a}$. In Newman-Penrose [8] notation, the Weyl tensor has components

$$
\Psi_{0}=b^{2}, \quad \Psi_{1}=0, \quad \Psi_{2}=\frac{1}{3} a b, \quad \Psi_{3}=0, \quad \Psi_{4}=a^{2},
$$

which is type $\mathrm{D}$ in the Petrov classification and the Maxwell tensor, given by (2.10), has components

$$
\Phi_{0}=b, \quad \Phi_{1}=0, \quad \Phi_{2}=a .
$$

\section{Collision of Light-Like Signals}

To demonstrate that the space-time with line element (2.7) and the Maxwell field (2.10) describe the gravitational and electromagnetic fields following the head-on collision of two homogeneous, plane, light-like signals, each composed of an electromagnetic shock wave accompanied by a gravitational shock wave, we replace $u, v$ in the argument of the cosine in (2.7) by $u_{+}=$ $u \vartheta(u), v_{+}=v \vartheta(v)$ where $\vartheta(u)$ is the Heaviside step function which is equal to unity for $u>0$ and is zero for $u<0$ (and similarly for $\vartheta(v)$ ) so that the line element we now consider reads

$$
d s^{2}=-\cos ^{2}\left\{\sqrt{2}\left(a u_{+}-b v_{+}\right)\right\} d x^{2}-d y^{2}+2 d u d v .
$$

Writing this line element in the form (2.8) with basis 1 -forms now given by $\vartheta^{1}=\cos \left\{\sqrt{2}\left(a u_{+}-b v_{+}\right)\right\} d x, \vartheta^{2}=d y, \vartheta^{3}=d v, \vartheta^{4}=d u$ we find that the components $R_{a b}$ of the Ricci tensor on the tetrad defined by this basis of 1-forms vanish except for

$$
\begin{aligned}
& R_{11}=-4 a b \vartheta(u) \vartheta(v), \quad R_{33}=b \sqrt{2} \delta(v) \tan \left(\sqrt{2} a u_{+}\right)-2 b^{2} \vartheta(v) \\
& R_{34}=2 a b \vartheta(u) \vartheta(v), \quad R_{44}=a \sqrt{2} \delta(u) \tan \left(\sqrt{2} b v_{+}\right)-2 a^{2} \vartheta(u)
\end{aligned}
$$


This Ricci tensor can be written in the form

$$
R_{a b}=\Lambda g_{a b}+2 E_{a b}+S_{a b},
$$

with $\Lambda=2 a b \vartheta(u) \vartheta(v), E_{a b}$ the tetrad components of the electromagnetic energy-momentum tensor calculated with the Maxwell field given by the 2-form

$$
F=b \vartheta(v) \vartheta^{3} \wedge \vartheta^{1}+a \vartheta(u) \vartheta^{1} \wedge \vartheta^{4}
$$

and $S_{a b}$ are the components of the surface stress-energy tensor [11] concentrated on the portions of the null hypersurfaces $u=0, v>0$ and $v=0, u>0$ and given by

$$
S_{a b}=b \sqrt{2} \delta(v) \tan \left(\sqrt{2} a u_{+}\right) \delta_{a}^{3} \delta_{b}^{3}+a \sqrt{2} \delta(u) \tan \left(\sqrt{2} b v_{+}\right) \delta_{a}^{4} \delta_{b}^{4} .
$$

We emphasize that in the post collision domain $(u>0, v>0)$ the field equations (3.3) can be written in the form

$$
R_{a b}-\frac{1}{2} g_{a b} R=T_{a b}+2 E_{a b} \quad \text { with } \quad T_{a b}=-2 a b g_{a b}
$$

where $R$ denotes the Ricci scalar. While the term $T_{a b}$ on the right hand side here has the form of a cosmological constant term it is equivalent to the energy-momentum-stress tensor for a perfect fluid for which the sum of the matter proper density and the isotropic pressure vanishes.

The Newman-Penrose components of the Maxwell field (3.4) are thus

$$
\Phi_{0}=b \vartheta(v), \quad \Phi_{1}=0, \quad \Phi_{2}=a \vartheta(u),
$$

while the Newman-Penrose components of the Weyl tensor are

$$
\begin{aligned}
& \Psi_{0}=-\frac{1}{\sqrt{2}} b \delta(v) \tan \left(\sqrt{2} a u_{+}\right)+b^{2} \vartheta(v), \quad \Psi_{1}=0 \\
& \Psi_{2}=\frac{1}{3} a b \vartheta(u) \vartheta(v) \\
& \Psi_{3}=0, \quad \Psi_{4}=-\frac{1}{\sqrt{2}} a \delta(u) \tan \left(\sqrt{2} b v_{+}\right)+a^{2} \vartheta(u) .
\end{aligned}
$$

On account of the appearance of the trigonometric functions in (3.5) and (3.8) we see that the coordinate $u$ has the range $0 \leq u<\pi / 2 \sqrt{2} a$ on $v=0$ and the coordinate $v$ has the range $0 \leq v<\pi / 2 \sqrt{2} b$ on $u=0$. Such restrictions are also exhibited in the Bell-Szekeres [1] solution and are discussed in [9].

We are now in a position to interpret physically what these equations are describing. First we consider the region of space-time corresponding 
to $v<0$. Now $R_{a b}=2 E_{a b}$ with $E_{a b}$ constructed from the Maxwell field $a \vartheta(u) \vartheta^{1} \wedge \vartheta^{4}$. All Newman-Penrose components of the Weyl tensor vanish except $\Psi_{4}=a^{2} \vartheta(u)$. We have here a solution of the vacuum EinsteinMaxwell field equations for $u>0$ corresponding to an electromagnetic shock wave accompanied by a gravitational shock wave, each having propagation direction $\partial / \partial v$ in the space-time with line element

$$
d s^{2}=-\cos ^{2}\left\{\sqrt{2} a u_{+}\right\} d x^{2}-d y^{2}+2 d u d v .
$$

The wave amplitudes are simply related via the parameter $a$, which could be thought of as a form of "fine tuning". We note that the space-time is flat and the fields vanish if, in addition to $v<0$, we have $u<0$. A similar situation arises in the region of space-time corresponding to $u<0$ with the gravitational shock wave described by $\Psi_{0}=b^{2} \vartheta(v)$ and the electromagnetic shock wave described by $b \vartheta(v) \vartheta^{3} \wedge \vartheta^{1}$, each having now propagation direction $\partial / \partial u$ in the space-time with line element

$$
d s^{2}=-\cos ^{2}\left\{\sqrt{2} b v_{+}\right\} d x^{2}-d y^{2}+2 d u d v .
$$

The wave amplitudes are again "fine tuned" via the parameter $b$. The electromagnetic and gravitational fields are non-vanishing in the region $v>0$ and vanish in the flat region $v<0$. After these two light-like signals collide at $u=v=0$ we obtain the post-collision region of space-time $u \geq 0, v \geq 0$. Clearly the subset $u>0, v>0$ is given by the Cartesian product space-time described in Section 2. This space-time includes a cosmological constant which has been considered in some works [10] as a possible candidate for dark energy and appears here as a consequence of the collision. On the boundary $u=0, v>0$ of this region we see from (3.5) that there is a light-like shell of matter with this boundary as history in space-time (a 2-plane of matter traveling with the speed of light, for example [11]) and from the last equation in (3.8) there is an impulsive gravitational wave with this boundary as history in space-time. Similarly the boundary $v=0, u>0$ is the history in spacetime of a light-like shell of matter following from (3.5) and of an impulsive gravitational wave following from the first equation in (3.8). These products of the collision, the light-like shells, the impulsive gravitational waves, the cosmological constant, can be thought of as a complicated redistribution of the energy in the incoming light-like signals. Such phenomena occur in most collisions involving thin shells, impulsive waves and shock waves, and are a consequence of the interactions between matter and the electromagnetic and gravitational fields [11]. Additionally one can have black hole production from the collision of two ultra-relativistic particles [12], the mass inflation phenomenon inside a black hole [13, 14] and the production of radiation from the collision of shock waves [15], [16]. 


\section{References}

[1] P. Bell and P. Szekeres, Gen. Rel. Grav. 5, 275 (1974).

[2] B. Bertotti, Phys. Rev. 116, 1331 (1959).

[3] I. Robinson, Bull. Acad. Polon. 7, 351 (1959).

[4] J. L. Synge and A. Schild, Tensor Calculus (University of Toronto Press, 1969).

[5] C. Barrabès and P. A. Hogan, Progress of Theor. Physics 126, 1 (2011).

[6] C. Barrabès and P. A. Hogan, Advanced General Relativity: Gravity Waves, Spinning Particles and Black Holes, International Series of Monographs on Physics no.160 (Oxford University Press, Oxford, 2013).

[7] H. Nariai, Gen. Rel. Grav. 31, 963 (1999), reprinted from Reports of Tohoku University (1951).

[8] E. T. Newman and R. Penrose, J. Math. Phys. 3, 566 (1962).

[9] C. J. S. Clarke and S. A. Hayward, Class. Quantum Grav. 6, 615 (1989).

[10] P. J. E. Peebles and B. Ratra, Rev. Mod. Phys. 75, 559 (2003).

[11] C. Barrabès and P. A. Hogan, Singular Null Hypersurfaces in General Relativity (World Scientific, Singapore, 2004).

[12] D. M. Eardley and S. B. Giddings, Phys. Rev. D66, 044011 (2002).

[13] E. Poisson and W. Israel, Phys. Rev. D41, 1796 (1990).

[14] C. Barrabès, W. Israel and E. Poisson, Class. Quantum. Grav. 7, L273 (1990).

[15] C. Herdeiro, M. O. P. Sampaio and C. Rebelo, JHEP 1107, 121 (2011).

[16] F. S. Coelho, C. Herdeiro and M. O. P. Sampaio, Phys. Rev. Lett. 108, 181102 (2012). 